\author{
E. Dahl-Jensen*), N.T. Doble**) and A.J. Herz \\ CERN, Geneva
}

A. Casanova***) and M. Gailloud***)

Institut de Physique Nucléaire de l'Université de Lausanne

A pulsed-transformer (flux-concentrator) magnet has been built and used in which the high-field region can be shaped according to experimental requirements. Access to the high-field region is easily provided both parallel to the field direction and in a plane normal to it. The design is such that the magnet can be dismantled completely, repaired or modified, and reassembled, even after having been operated for several thousand pulses. Two such magnets have been used in an experiment to measure the magnetic moment of the $\Lambda^{0}$ hyperon; each produced $200 \mathrm{kG}$ in a volume of $200 \mathrm{~cm}^{3}$ for about 5000 pulses and is still in usable condition.

Submitted to Nuclear Instruments and Methods

Geneva, June 1969

*) Now at the Niels Bohr Institute; Universit.y of Copenhagen.

**.) Now at the Max-Planck-Institut für Physik, Munich.

***) Supported in part by the Swiss National Fund. 
1. PRINCIPLES OF THE MAGNET DESIGN

The magnet we describe is essentially a pulsed trans rmer combining a single-turn secondary with a multiturn primary. The basic principles of this type of magnet have been discussed in the literature ( ref. 1 and 2, for example). We recall that in general the secondary consists of a single block of conductor containing a central bore which is linked radially to the outside of the block by means of a slot as illustrated in Fig. 1. When a current pulse is passed through the primary windings a current is induced in the opposite sense in the outer surface of the secondary. This current, which is constrained by the skin effect, is forced to flow along the faces of the slot and round the central bore, producing a magnetic field in it. Alternatively, the magnetic flux produced by the primary current may be regarded as being concentrated in the bore of the secondary (Fig. 1), where the radial slot serves to prevent the latter from being short-circuited. For this reason such a magnet is commonly referred to as a "flux concentrator".

A magnet of this type has certain features which are of advantage in the production of high pulsed magnetic fields. The principal forces are exerted on a single block of metal, thus the magnet does not depend critically on the resistance to stress of relatively weak insulating materials*). The inductance presented to the external circuit can be adjusted by choosing the number of primary turns. Furthermore, a field of a given configuration may be produced by shaping the inside surface of the secondary, since the lines of flux are effectively bounded by the current flowing in this surface. The principal disadvantage of this type of magnet is the low efficiency with which energy is transferred into high magnetic field in the useful volume of the magnet (see Table I).

\subsection{General features of the magnet}

The pulsed-transformer magnets described in the literature (for example ref. 1 -6) possessed a central bore of circular cross-section into which the magnetic flux was concentrated. The magnet we describe represents a more

*) In fact the electromagnetic forces acting on the primary windings are small if these windings are embedded in a helical groove cut into the outside of the secondary. 
general application of the principles of flux concentration. It is essentially a "double" or "twin" flux concentrator built up of two essentially identical halves which are in contact in a plane of mirror symmetry and have their primary windings connected in series ( $F$ igs $2 a$ and $2 b$ ). The plane of symmetry divides the central high-field region in two, and all field lines cross it normally. This type of design makes it relatively easy to give complex shapes to the high-field region, and to provide access at right angles to the field by cutting channels of appropriate shape into the faces of contact between the two half blocks. If charged-particle beams are introduced through such channels, they will remain in the contact plane and can be made to leave again through channels in the same plane after having been deflected by the field.

The general principles of the mechanical design, described in detail in Section 2 below, were

(i) It must be possible to dismantle the magnet for inspection, repairs or modifications and to reassemble it subsequently without major difficulties.

(ii) The primary windings and their high-voltage insulation must not be submitted to large mechanical forces during pulsing. The forces were taken in part by the secondary blocks and in part by constraining yokes clamped around them.

\subsection{Specific application of the magnet}

The features of the magnet outlined above have been exploited in an experiment to determine the magnetic moment of the $\Lambda^{\circ}$ hyperon.

This type of experiment relies for the production of the hyperons on the interaction of an intense pion beam with the nuclei of a target placed inside the magnet. The hyperons traverse the high field produced in the magnet, and their decays are detected in pellicles of nuclear emulsion also located inside the magnet. In order to make a precise measurement of the $\Lambda^{0}$ magnetic moment by this method, it is necessary to impose a field in excess of $200 \mathrm{kG}$ in a direction transverse to the flight paths of the hyperons and over a length of 10 to $12 \mathrm{~cm}$. Moreover, a layout which allows the detection of the hyperons both to the left and to the right of the primary beam helps to eliminate biases as well as to increase the number of events. 
The high-field bore, which in each half of the secondary contains the path of the hyperons from the target to the detectors, is shaped as a narrow oblong corridor, oriented so as to form an extension of the slot of the flux concentrator ( $F$ ig. 2(a)). In the experiment considered, this serves as a collimator to shield the detectors from some of the charged particles originating from the target.

Finally, we required that the magnet should withstand operation for of the order of $10^{4}$ pulses.

The magnet was built to satisfy these conditions. Its electrical characteristics matched to a $75000-\mu \mathrm{F}$ condenser bank ${ }^{7 \text { ) }}$ are set out in Table I (p. 3a).

\section{DESIGN OF THE MAGNET}

The principal features of the design of the magnet are described below. Some stages in its assembly are further illustrated in Fig. 2.

\subsection{Primary}

The primary windings consists of four spirals of copper, two of smaller and two of larger diameter, each having four turns and the same pitch and rectangular cross-section. Each spiral was bent from a bar of the material and afterwards turned to its final dimensions. A groove of square cross-section was turned into the outside of each spiral to receive a copper tube of $5 \mathrm{~mm}$ bore (Fig. $2(c)$ ). This provides a passage for cooling water.

\subsection{Secondary}

The secondary consists of two identically-shaped blocks, made of Cu-Cr-Zr alloy ${ }^{*}$. Each block is machined out of a thick circular disc, into the outer surface of which a deep helical groove is turned to receive the primary windings (see paragraph 2.3). The insides are turned and milled out to form the "corridor" (Fig. 2), through which the magnetic flux threading the coil is concentrated. Cylindrically-shaped cavities in one end of each corridor provide room for the particle detectors (nuclear-emulsion pellicles). The slot

*) The alloy of $\mathrm{Cu}$ with $\sim 1 \% \mathrm{Cr}_{\mathrm{r}}$ and $\sim 0.1 \%$ Zr has an electrical resistivity $\left(2.1 \times 10^{-6} \mathrm{Ohm} / \mathrm{cm}\right)$ which is only about $15 \%$ higher than that of pure copper. Its tensile strength $\left(50-60 \mathrm{~kg} / \mathrm{mm}^{2}\right)$ is, however, about twice that of copper. The presence of zirconium in this alloy, as manufactured by Dsnabrücker Kupfer-und Drahtwerk, Germany, leads to increased resistance to creep. 


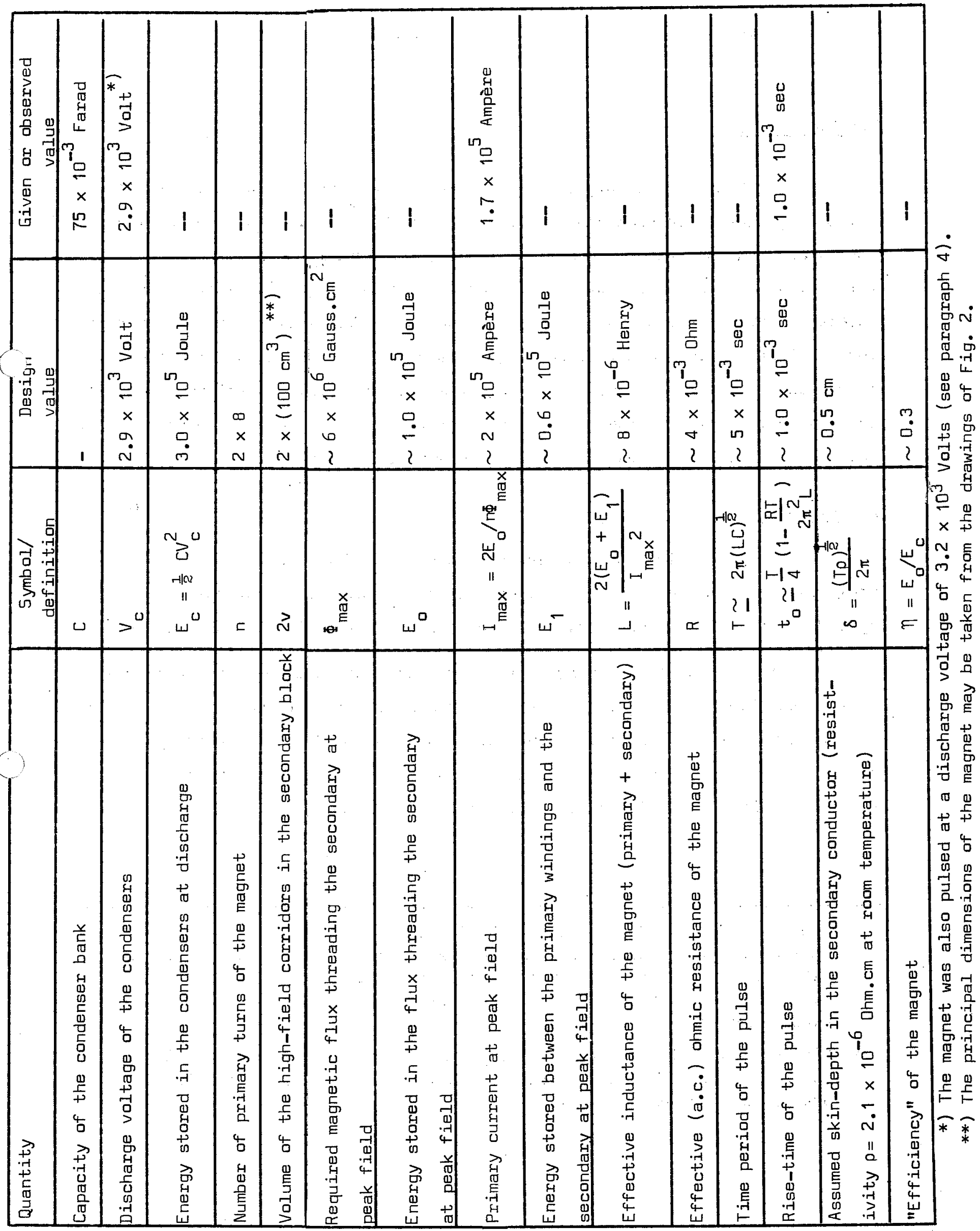


of the flux concentrator is filled with a spacer of Vetronite ${ }^{*}$ ).

Cooling water is circulated through four holes traverșing the secondary blacks.

\subsection{Insulation}

Each of the primary spirals is individually encased with layers of Vetronite, glued into place and sealed with Araldite ${ }^{* *}$, in order to provide insulation that withstands a tension of $3 \mathrm{kV}$. The bottom and sides of the helical groove in each of the two secondary blocks are similarly lined with layers of Vetronite and the insulation tested to $3 \mathrm{kV}$. Finally, the two spirals (one smaller and one larger) belonging to each half of the magnet are screwed into place in the secondary, making use of a silicone fluid to act as lubricant and insulating filler between the sliding surfaces (Fig. 2(c)). Each half of the coil thus completed is tested to withstand a tension of $6 \mathrm{kV}$.

\subsection{Assembly and connections}

The two halves of the secondary are clamped together by twelve stainiless-steel bolts. This is necessary to ensure elastic behaviour of the system although the forces between the halves are attractive during a pulse.

The ends of the primary spirals are connected together at the junction between the two coil halves (Fig. 2) in such a manner that the primary circuit is formed by the outer spiral of the first half followed by the inner spiral of the second half, then linked by an external bridge to the inner spiral of the first half and completed by the outer spiral of the second half.

*) "Vetronite EPG11" in the form of laminated sheets, consisting of fibreglass impregnated with epoxy resin, is manufactured by Isola Werke, Breitenbach, Switzerland. In order to achieve satisfactory insulation and mechanical behaviour it was found necessary to have the Vetronite sheets ground to close tolerances.

**) "Araldite AW 106" is a liquid epoxy resin which gives a bond of high resistance to impact and vibration. It is manufactured by CIBA A.G., Basel, Switzerland. 
Current is supplied to the coil via six coaxial cables from the condenser bank which are attached to a collecting bar. Reversal of the orientation of the magnetic field through the coil can be effected by reversing the connections to the primary windings of this collecting-bar assembly.

\subsection{Constraining yoke}

It became evident from tests of prototypes that the secondary block of a magnet of this type is not able, without external constraint, to withstand the forces generated in the central volume by a field in excess of $200 \mathrm{kG}$. A yoke was therefore designed to fulfil the following functions: to prestress the secondary in the direction perpendicular to the plane of the slot, so that the forces generated across this plane during a pulse would be taken up. without causing the slot to open permanently; and to constrain the primary windings against the radial forces which tend to eject them fram the secondary.

This yoke consists essentially of two heavy rings of stainless steel, one clamped around each half of the coil. In fact, each ring is turned to the external diameter of the coil; it is then split horizontally into two halves which are mounted and pulled together around the coil by the application of a pair of heavy steel clamps. Each of these clamps bears on the two halves of the ring via wedge-shaped pads and is drawn into place with the aid of bolts. At the side of the coil containing the slot it is necessary to insulate the two halves of the ring from each other. This is done by means of a layer of Vetronite between the pads and under the heads of the bolts holding the corresponding clamp in place.

Finally, the coil with its constraining yoke is mounted between two rectangular insulating end pieces. The overall weight of the magnet assembly is about 1 ton.

\section{PERFORMANCE OF THE MAGNET}

\subsection{Magnetic field}

Calibrated search coils connected to an integrating circuit were used to establish the magnitude and direction of the magnetic field generated in the magnet.

The time variation of the magnitude of the field $|\vec{H}|$ during a pulse 
is recorded in Fig. 3. The observed rise time of the field of approximately $1 \mathrm{~ms}$ is in accordance with the expected value given in Table I.

The variation of the magnetic field $(|\vec{H}|)$ with position inside the magnet is illustrated in Fig. 4. This shows the distribution of the lines of flux in the horizontal median plane of the magnet (Fig. 2(b)). The component of the field normal to this plane is, by symmetry, zero at all points in this plane; it was also measured to be comparatively small $\lesssim 5 \%$ of the total field) at all points within the central high-field corridor. The distribution of the field is, as expected, symmetric about the vertical plane of contact between the two halves of the magnet. Some penetration of the copper alloy by the lines of flux is permitted by the finite thickness of the current "skin" around the central corridor. This effect is a function of the local temperature and hence of the current density in the conductor as well as of the time period of the pulse. As the discharge voltage of the condenser bank is increased one may, therefore, expect the magnetic field to penetrate more deeply into the metal.

In Fig. 5(a) we plot, for discharge voltages of $0.94,1.88,2.90$ and $3.20 \mathrm{kV}$, the absolute magnitude of the field $(|\vec{H}|)$ recorded as a function of distance along the lines $T D$ and $T D^{\prime}$ in the magnet ( $F$ ig. 4). These lines correspond to the mean flight paths of the $\Lambda^{\circ}$ hyperons in our experiment. The variation of the magnitude of the field at the points $P$ and $P^{\prime}$ of maximum field is shown in Fig. $5(\mathrm{~b})$ as a function of discharge voltage. Note that the intensity of the field does not increase linearly with voltage. This effect is, in part at least, due to the increase in skin depth mentioned above, but other effects are likely to contribute also.

We may summarize the conditions obtained for the experiment by quoting below the cross-product of the mean field $(\vec{H})$ with the path length $(\ell)$ of the hyperons at the two values of the condenser discharge voltage $\left(V_{e}\right)$ used:
$v_{c}(k V)$
$|\vec{H}| \times \vec{\ell}$

2.90

$192 \mathrm{kG} \times 11 \mathrm{~cm}$

3.20

$208 \mathrm{kG} \times 11 \mathrm{~cm}$ 


\subsection{Mechanical behaviour}

The experiment to determine the $\Lambda^{\circ}$ magnetic moment was carried out with two similar coils of the type we have described.

During use the magnets were subjected to repeated pulsing at a repetition rate of approximately 1 pulse every 20 seconds for some hundreds of pulses at a time. In these conditions the maximum temperature of the nuclear emulsions placed inside the magnets to detect the $\Lambda^{\circ}$ hyperons was observed to be $40^{\circ} \mathrm{C}$.

In the full course of the experiment one of the coils received a total of about 5000 pulses at a condenser discharge voltage of $2.9 \mathrm{kV}$. A single breakdown of the insulation occurred at the junction of the primary windings after about 3000 pulses, the repair of which entailed the complete reinsulation of the coil. The other coil withstood, without major breakdown, about 4000 pulses at $2.9 \mathrm{kV}$ and about 1000 pulses at $3.2 \mathrm{kV}$.

In the secondary blocks of each coil, however, cracks were observed to develop in the region around the target. This is the region of greatest mechanical strain during pulsing, and it is also the region in which the highest temperatures $\left(200^{\circ} \mathrm{C}\right.$ during pulse) have been observed. With continued pulsing the cracks grew slowly. The appearance and growth of the cracks was not associated with major changes in the magnetic field or field distribution; only in their neighbourhood was a relatively slight $(\leqslant 5 \%)$ decrease in field recorded.

We conclude that it is possible to constrain a coil of this type sufficiently to prevent rapid deterioriation and failure due to the large-scale effects of the forces tending to open the slots in the secondary blocks. The principal mechanical limitations to the field that can be generated appear to be the local stresses to which the material of the secondary is subjected in the regions of maximum strain and maximum flux concentration. 


\section{ACKNOWLEDGEMENTS}

The work described in this paper was carried out within the framework of the collaboration to measure the $\wedge^{\circ}$ magnetic moment in which the emulsion groups at Ankara, CERN, Lausanne, Munich and Rome took part. We should like to acknowledge the support of our colleagues and members of the collaboration who contributed to the realization of the project.

The development of the pulsed-transformer magnet owes much to the pioneering work of Drs M. Morpurgo and L. Hoffmann. The magnets were built in the Central Workshop of CERN. The active participation of the leaders of this workshop and the meticulous work of their personnel, and especially of Mr P. Loosli, have greatly contributed to their successul functioning. Thanks are due also to Mr R. Lorenzi and Mr M.A. Roberts for their suggestions and help in the construction and operation of the equipment.

Finally we should like to express our appreciation to the authorities in charge of the collaborating laboratories who supplied support for the project. 


\section{REFERENCES}

1) H.P. Furth, M.A. Levine and R.W. Waniek, Rev. Sci. Instrum. 28, 949 (19.57).

2) Y.B. Kim and E.D. Platner, Rev. Sci. Instrum. 30, 524 (1959).

3) B. Howland and 5. Foner, Proc. Int. Conf. on High Magnetic Fields at MIT (1961), (MIT Press and Wiley Inc., 1962), 249.

4) L. Hoffmann and V. Scheuing, "Transformer coils for high magnetic fields", CERN $63-36(1963)$.

5) M.N. Wilson and K.D. Srivastava, Rev. Sci. Instrum. 36, 1096 (1965).

6) H. Brechna, D.A. Hill and B.M. Bailey, Rev. Sci. Instrum. 36, 1529 (1965).

7) M. Morpurgo, L. Hoffmann and W.M. Gibson, "Design and construction of a 200-kGauss pulsed magnet", CERN 60-27 (1960); E. Braunersreuther, J.C. Combe, L. Hoffmann and M. Morpurgo, "A 200-kGauss pulsed magnet for emulsion experiments", CERN 62-7 (1962). 


\section{FIGURE CAPTIONS}

Fig. 1 Schematic representation of a pulsed-transformer magnet, showing the concentration of magnetic flux in the central bore of the secondary.

Fig. 2 Assembly of the two halves of the "twin" flux concentrator:

(a) Side elevation.

(b) Horizontal section (section AA of Fig. 2(a). T is the target, D and $D^{\prime}$ are the positions in which the nuclear-emulsion pellicles are placed.

(c) Detailed section showing the insulation between the primary windings and the secondary.

Fig. 3 Time variation of the field $(\vec{H}$ ) in the magnet.

Fig. 4 Magnetic field lines $(\vec{H})$ in the horizontal median plane of the magnet (section $A A$ of $F$ ig. 2(a)). TD and $T D^{\prime}$ represent the mean lines of flight of $\Lambda^{0}$ hyperons from target to detector.

Fig. 5 Variation of the magnetic field $(\vec{H})$ as a function of:

(a) distance $(x)$ along the lines TD, TD';

(b) condenser bank discharge voltage $\left(V_{c}\right)$, at the points $p$, $p^{\prime}$ of maximum field in the magnet. 


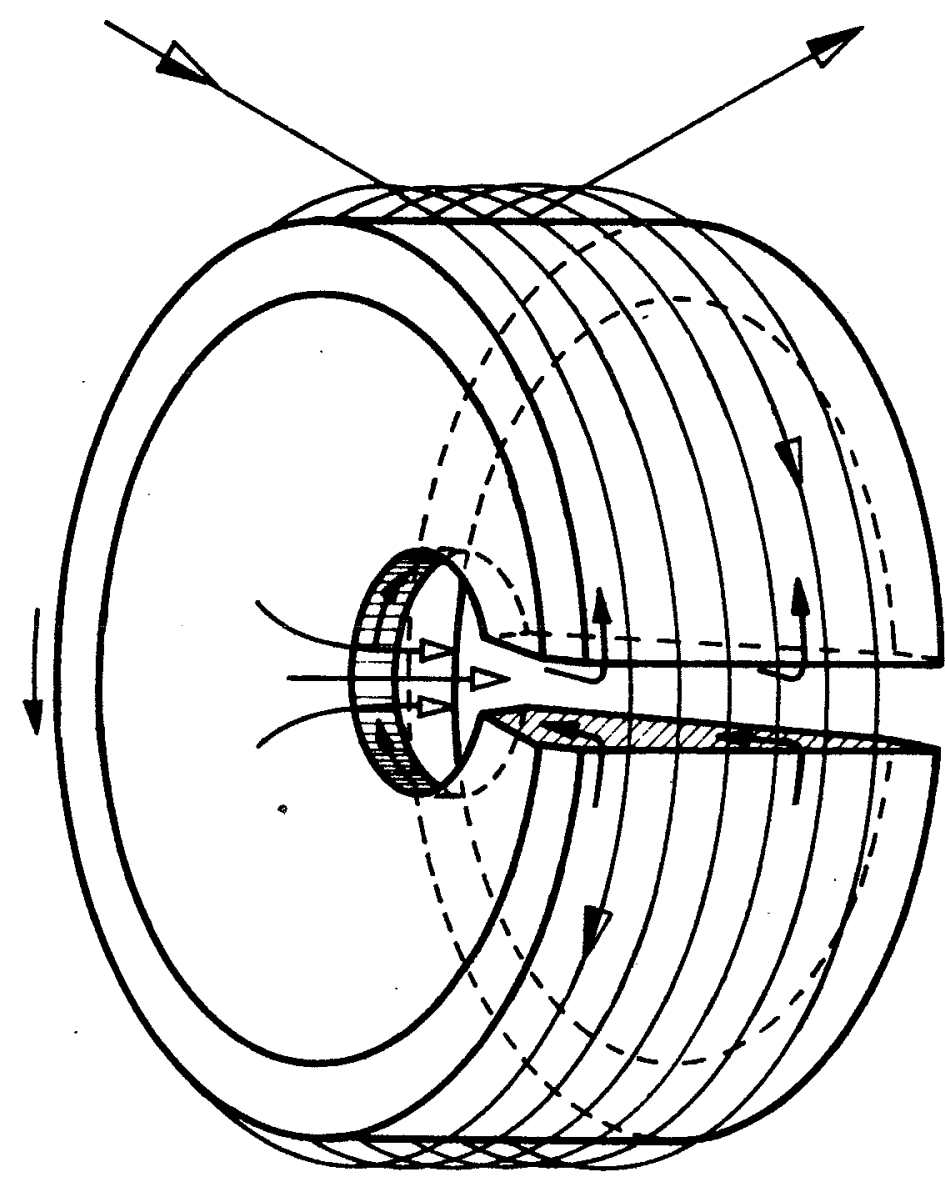

$\longrightarrow$ PRIMARY CURRENT

$\longrightarrow$ SECONDARY CURRENT

$\longrightarrow \quad$ MAGNETIC FIELD 
v
$\frac{0}{1}$

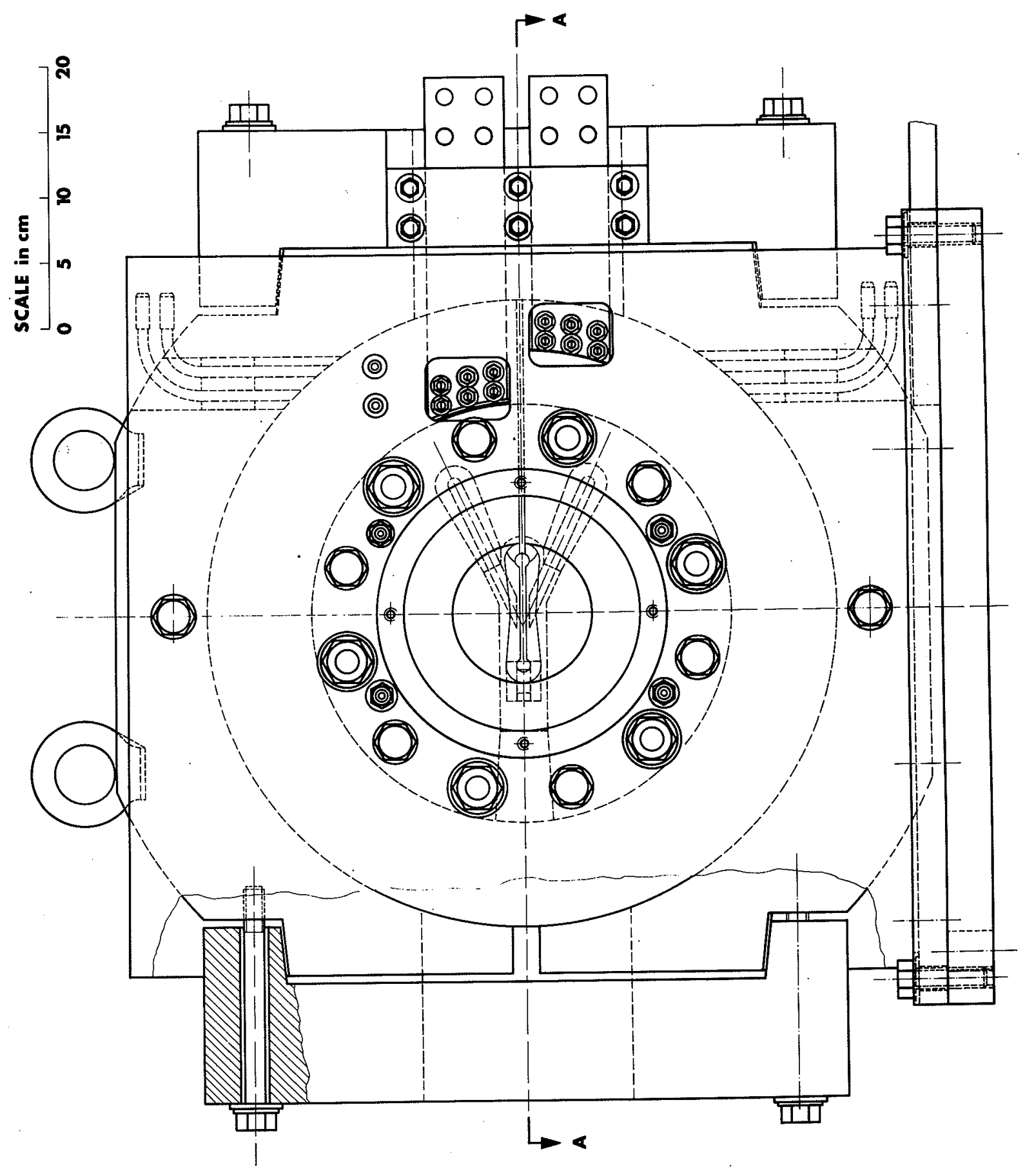




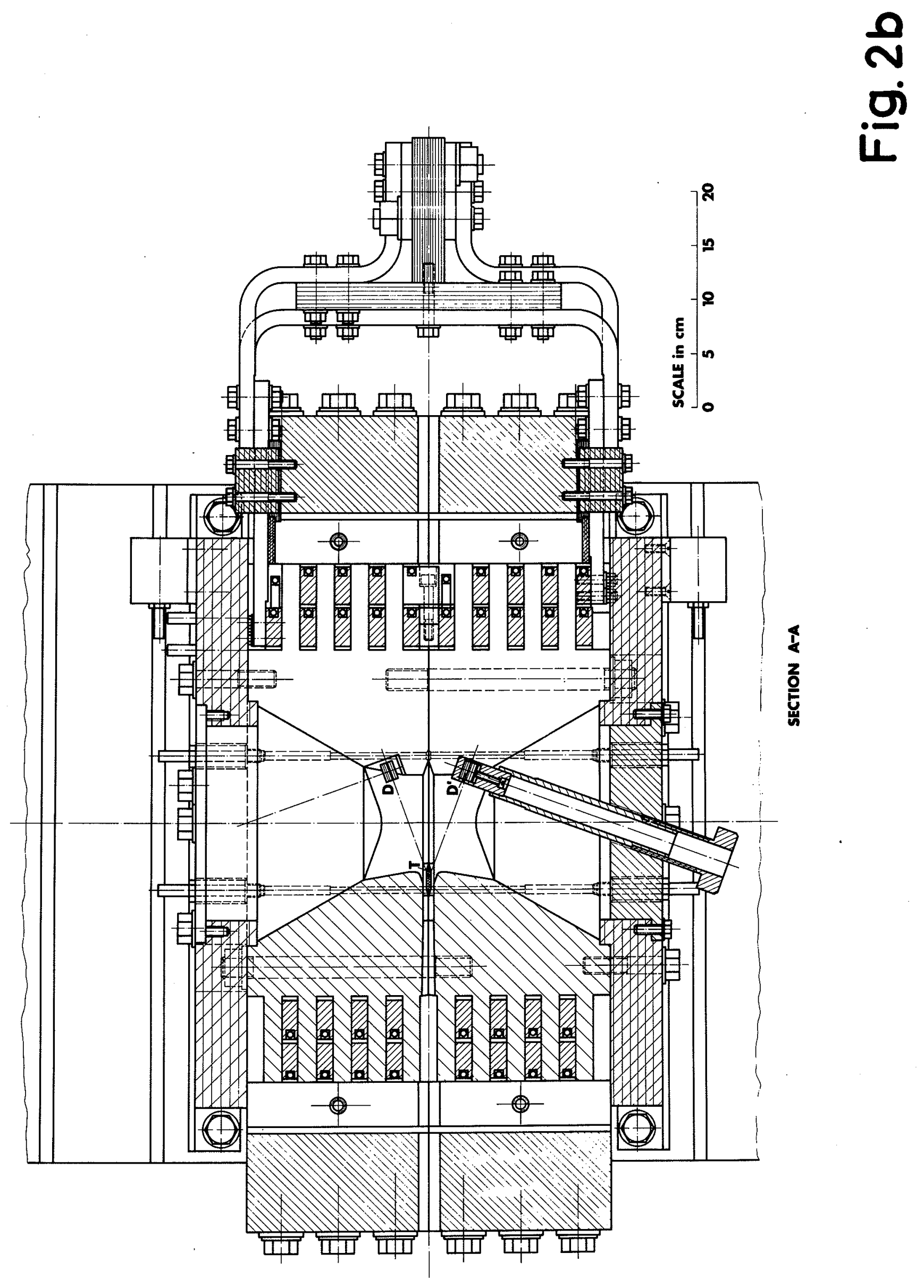




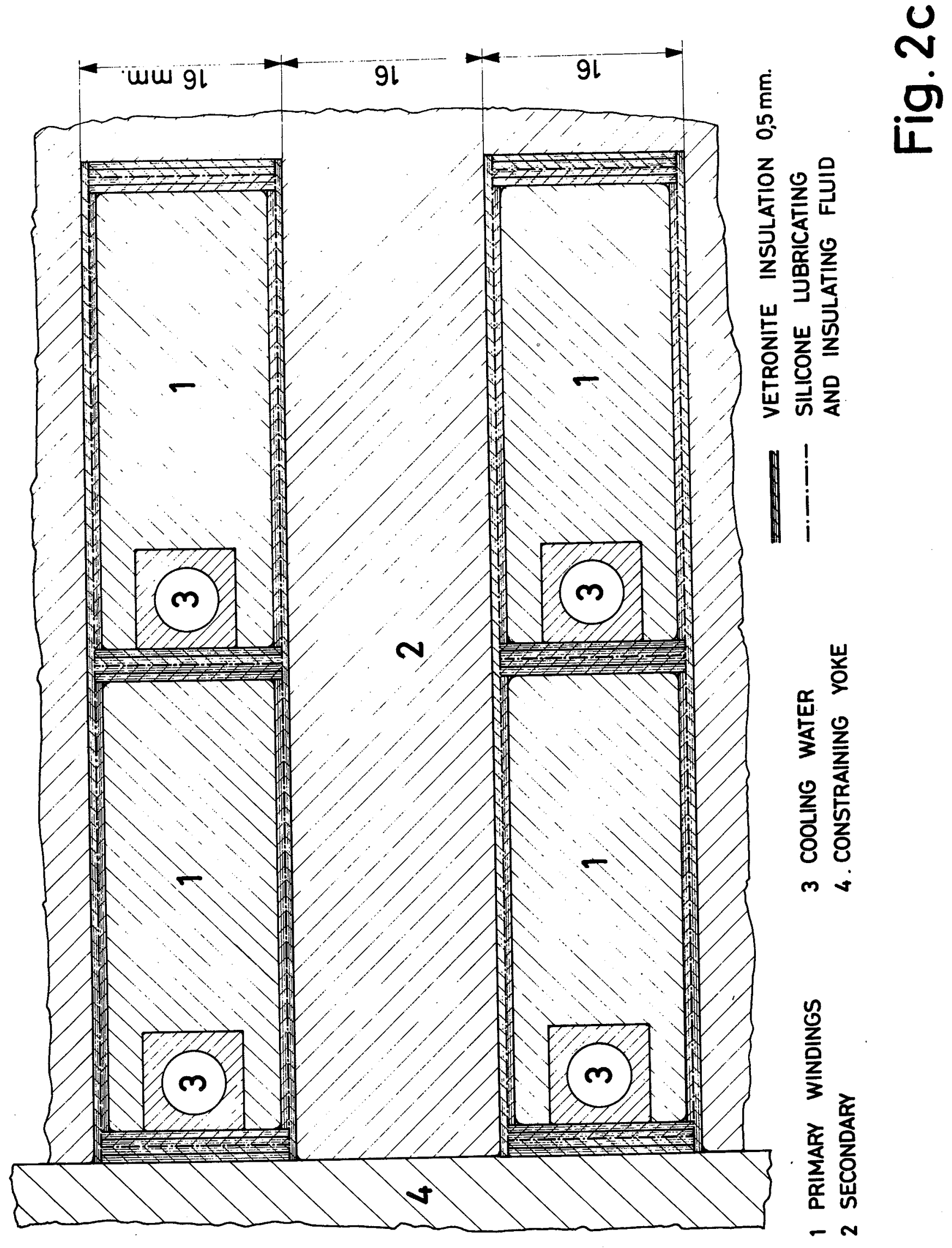




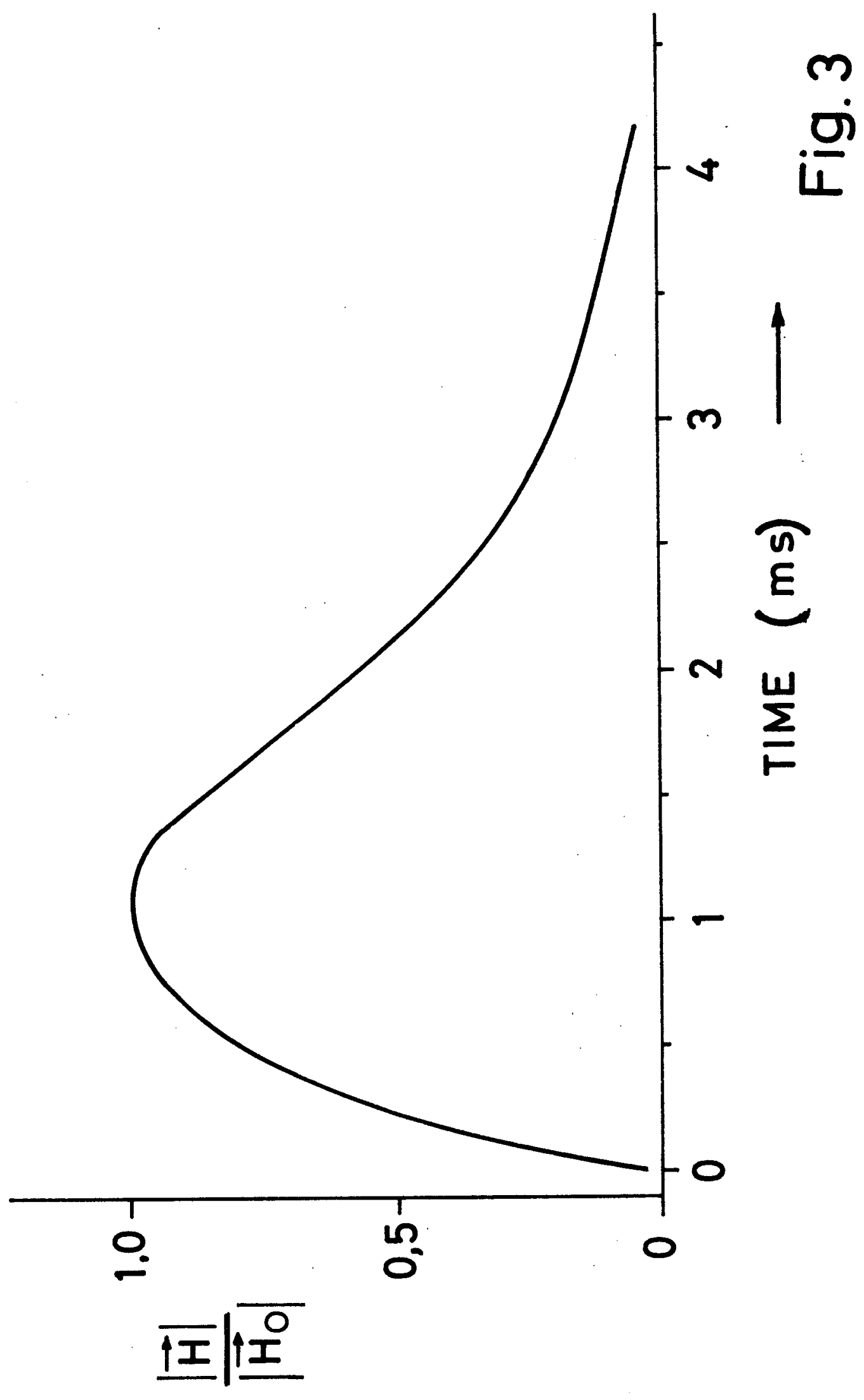




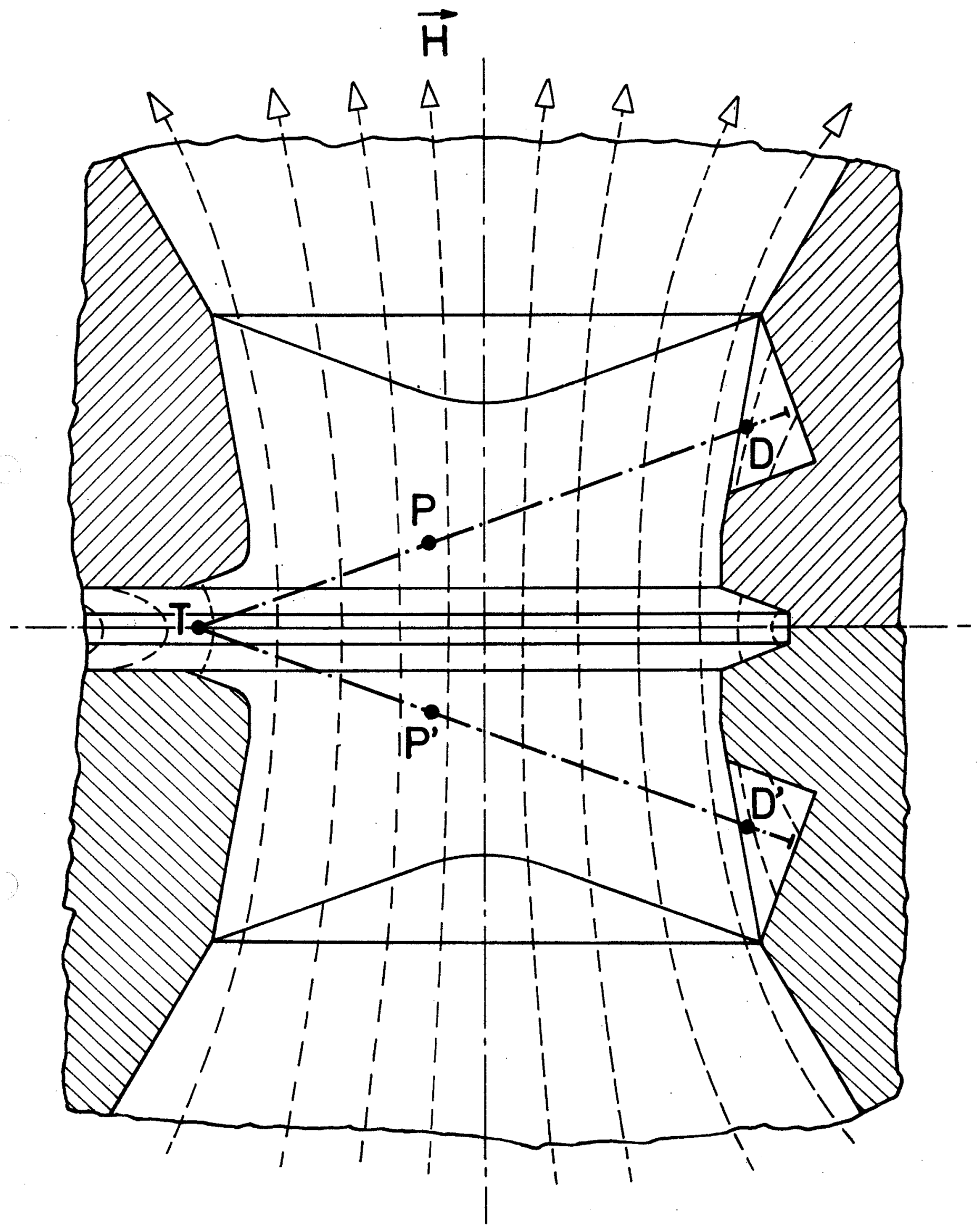


$|\vec{H}|$ kGauss

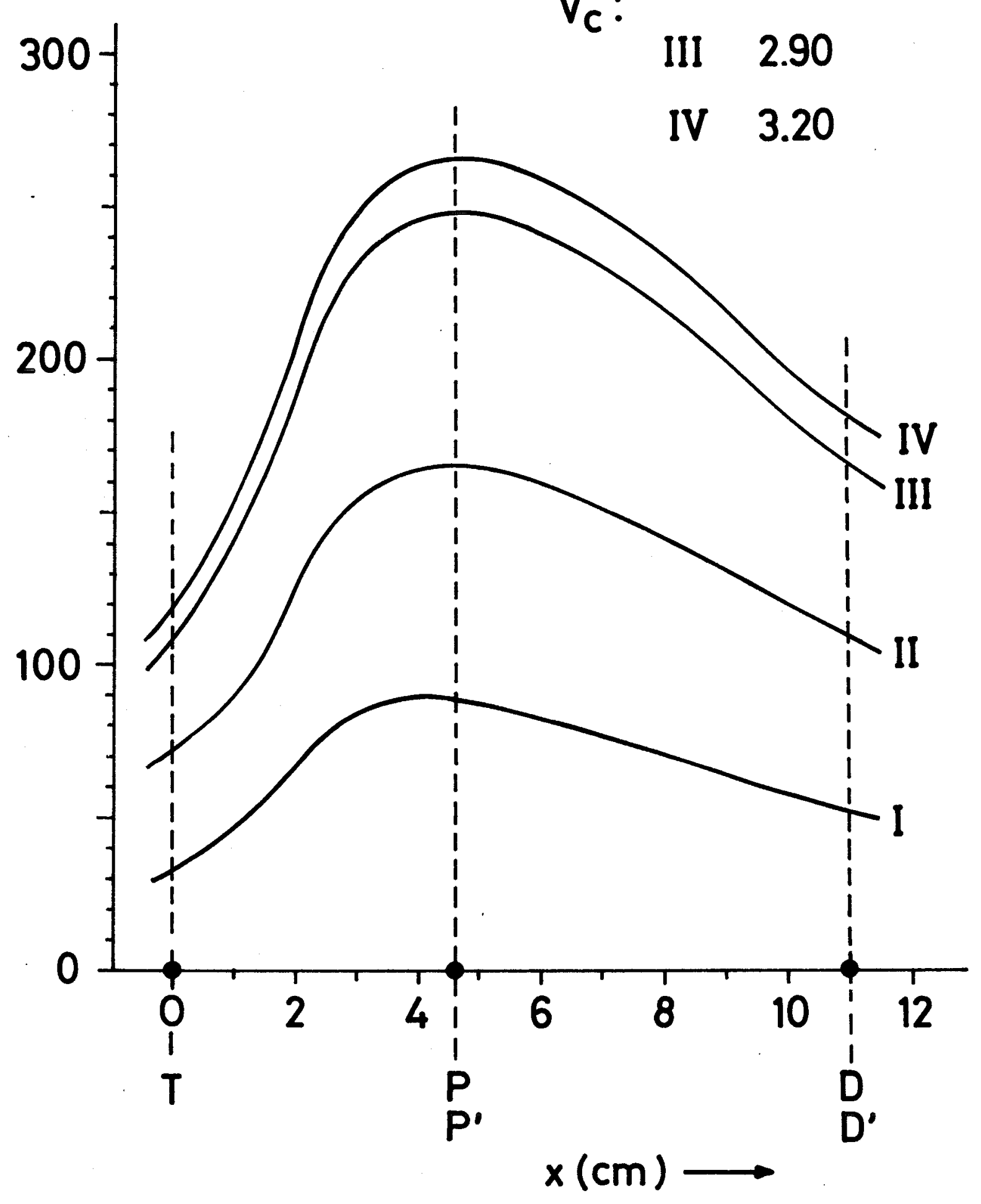

Fig. $5 a$ 
$|\vec{H}|$ kGauss

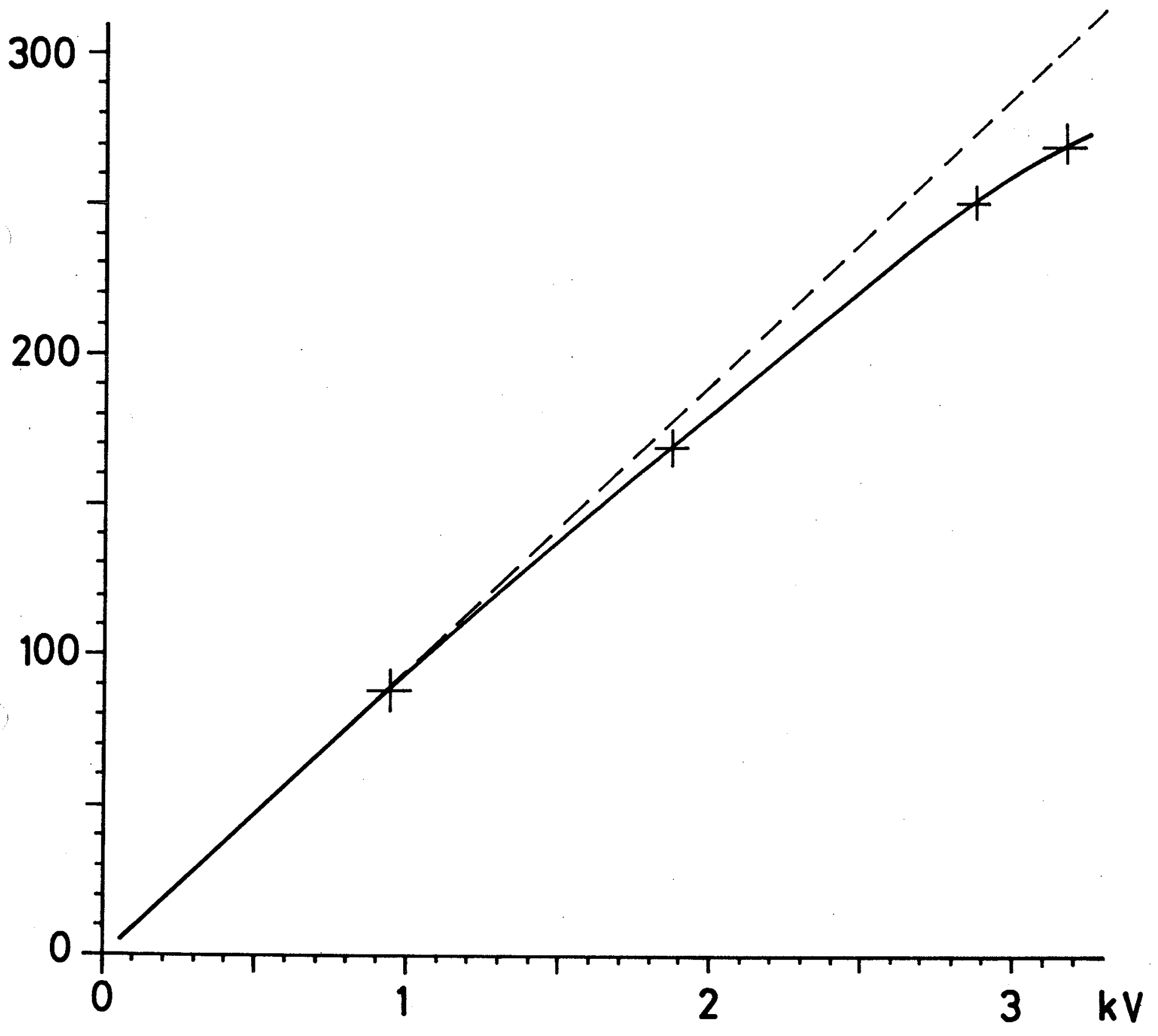

Fig. 5b 